\title{
Cellular Shear Adhesion Force Measurement and Simultaneous Imaging by Atomic Force Microscope
}

\author{
Yu Hou ${ }^{1,2}$, Zuobin Wang ${ }^{1 * 2}$, Dayou $\mathrm{Li}^{1,2^{*}}$, Renxi Qiu ${ }^{2}$, Yan $\mathrm{Li}^{1}$ and Jinlan Jiang ${ }^{3^{*}}$ \\ ${ }^{1}$ Changchun University of Science and Technology, CNM \& JR3CN, Changchun, China \\ ${ }^{2}$ University of Bedfordshire, JR3CN \& IRAC, Luton, UK \\ ${ }^{3}$ Jilin University, China-Japan Union Hospital, Changchun, China \\ *Corresponding authors: wangz@cust.edu.cn; dayou.li@beds.ac.uk; Jiangj12003@hotmail.com
}

\begin{abstract}
This paper presents a sensitive and fast cellular shear adhesion force measurement method using an atomic force microscope $(A F M)$. In the work, the $A F M$ was used both as a tool for the imaging of cells on the nano-scale and as a force sensor for the measurement of the shear adhesion force between the cell and the substrate. After the cell imaging, the measurement of cellular shear adhesion forces was made based on the different positions of the cell on the nano-scale. Moreover, different pushing speeds of probe and various locations of cells were used in experiments to study their influences. In this study, the measurement of the cell adhesion in the upper portion of the cell is different from that in the lower portion. It may reveal that the cancer cells have the metastasis tendency after cultured for 16 to 20 hours, which is significant for preventing metastasis in the patients diagnosed with early cancer lesions. Furthermore, the cellular shear adhesion forces of two types of living cancer cells were obtained based on the measurements of $A F M$ cantilever deflections in the torsional and vertical directions. The results demonstrate that the shear adhesion force of cancer cells is twice as much as the same type of cancer cells with TRAIL. The method can also provide a way for the measurement of the cellular shear adhesion force between the cell and the substrate, and for the simultaneous exploration of cells using the AFM imaging and manipulation.
\end{abstract}


Keywords: AFM cantilever, cellular shear adhesion force, nano-manipulation.

\section{Introduction}

Cell adhesion refers to cell-cell and cell-matrix interactions, which is not only a main approach for the communication between cells but also an important biological phenomenon for the maintenance of morphology and functions in multi-cellular organisms [1, 2]. For the research of artificial organs, the cell-cell adhesion and cellsubstrate adhesion are closely related to the biological compatibility of artificial implants. Meanwhile, the cellsubstrate adhesion is the key factor critical for the success of the implantation operation of artificial organs. Thus, great attention has been paid to the cell-cell adhesion, cell-matrix adhesion, related cell morphology structure, function, deformation ability and adhesion of cells [3, 4].

To understand the mechanical properties of single cells as well as their variations, it is necessary to establish a proper method for the measurement of cell-cell adhesion or cell-matrix adhesion in vivo and overcome the limitations of current methods [5]. There were several methods developed for the measurement of cell adhesion, including the mechanical method, parallel-plate flow chamber ( $P P F C$ ), cell isolation, micro-fluidic method, micropipette, optical tweezers and atomic force microscope $[6,8]$. The mechanical method evaluated the cell adhesion through calculating the percentage of tumor cells [9]. The PPFC and cell isolation methods detected cells in groups [10]. Currently, the micro-pipette and micro-fluidic methods make the very significant portions of cell adhesion measurements [11]. However, as a result of the complicated deformation features of cells, it is difficult to obtain the precise value of an adhesion force. Optical tweezers could manipulate cells accurately on the pN-scale. However, during the manipulation, cells could be injured due to the high-intensity laser beam [12].

The AFM is used as a tool for the imaging of cells on the nano-scale [13]. It can implement the mechanical detection of a single cell with high precision based on the optical lever, and obtain the cell morphology in real-time. The probe cantilever can detect the morphology character on the cell surface as a terminal executor. Meanwhile, as a manipulator, it can also acquire various mechanical properties on the cell surface. Applying lateral forces to characterize cell detachment forces by AFM has been investigated in numerous studies. They used the back of cantilevers to detach cells and characterize detachment forces and completely ignored the tip of probe [14, 16]. Hence, most of studies for the detection of the cell adhesion force with an AFM lacked methods to measure the shear adhesion force of living cells and obtain the morphology character at the same time. Moreover, the shear adhesion 
force was usually calculated by the deflection of cantilever with one direction. There might be some extent errors in the lateral direction and without including the effects of pushing speeds. Thus, in this work, a simple and sensitive method to measure the cellular shear adhesion force by AFM has been presented. The AFM was used both as a tool for the imaging of cells on the nano-scale and as a force sensor for the measurement of the shear adhesion force between the colon cancer cell and the substrate. The method displaced the cell using the AFM cantilever tip along the sample surface and measured the resulting deflections with the lateral signal and vertical signal of the cantilever. An optical feedback system based on a charge-coupled device (CCD) camera was used together with an AFM to manipulate cells in real-time, so that it was able to perform the morphology measurement of some specific targeted cells. Moreover, different pushing speeds of probe were the first used in experiments to study their influences. The experimental results have shown that the method can provide an effective way for the measurement of the horizontal shear adhesion force between the cell and substrate, and for the exploration of shear adhesion forces of cancer cells using both the AFM imaging and manipulation systems. The method is described in detail in the following sections.

\section{Materials and Methods}

\section{Cell culture}

The present studies were performed for the implementation of the tumor necrosis factor-related apoptosisinducing ligand (TRAIL)-induced apoptosis of cancer cells [17]. Hence, two types of cancer cells were observed and manipulated in the experiment, including SW480 human colon cancer cells and the same type of cancer cells with the immune expression of TRAIL. The SW480 human colon cancer cells were divided from the standard cell line with both the typical ability to invade to other tissues and be less prone to rupture due to the highly reactive molecules of cells. The different status of the same type of cancer cells can be used to verify the improved method for AFM based cell adhesion measurements. Those cells were passaged simultaneously in the endothelial cell basal medium (RPMI-1640) with $10 \%$ fetal bovine serum and cultured in a controlled environment of $5 \% \mathrm{CO}_{2}$ at $37.5^{\circ} \mathrm{C}$. The cells were seeded on a non-coated cover-slip and used for the experiment under the same condition. The final concentrations of TRAIL were 300ng/ml and 200ng/ml. The cancer cells and cells with TRAIL were cultured for 16 to 20 hours. Those cells were separated from the same batch, and cultured in the same conditions, so that the different topographies and shear adhesion forces could prove that the TRAIL did work on the cells. 


\section{Modeling of the manipulation of cells under pushing}

During the operation to manipulate cells, the nano-scale tip of AFM will suffer from the various forces such as the Van der Waals force, electrostatic force, capillary force, friction force, surface tension and Coulomb force. All of these forces can make the twist and bend deformations of the cantilever. A model for the deformation of the cantilever in three dimensions has been established for the forces along with the three orthogonal coordinate axes $\left(F_{x}, F_{y}\right.$ and $\left.F_{z}\right)$, as shown in Figure 1.

The cell manipulation task is implemented by the self-made AFM system, and the cellular shear adhesion force will act on the probe tip and make it bending due to the shear adhesion force between the cell and the substrate. When the torsional force of the cantilever is greater than the cellular shear adhesion force, the cell will be separated from the substrate. Thus, the cellular shear adhesion force can be obtained by the cantilever deflection.

In the components of the three forces along with the three orthogonal coordinate axes, the force $F_{x}$ will produce the twist of the cantilever around its central axis, and it can be expressed as [18]

$$
F_{x}\left(H+\frac{S}{2}\right)=k_{t} \theta
$$

where $H$ is the height of probe, $S$ is the thickness of cantilever, $k_{t}$ is the torsion spring constant of cantilever, and $\theta$ is the twist angle from the rest position. The torsion strength of cantilever is [19]

$$
k_{t}=G \beta w S^{3} / L
$$

where $G$ is the shearing elasticity modulus of the cantilever material, $w$ is the width of cantilever, $L$ is the length of cantilever, and $\beta$ is the constant calculated by S/w. Here, $L=450 \mu \mathrm{m}, H=18 \mu \mathrm{m}$ and $k_{t}=1.71 \times 10^{-7} \mathrm{~N} . \mathrm{m} / \mathrm{rad}$ can be obtained.

As shown in Figure 1B, a torque is generated on the cantilever by $F_{y 1}$ which is the component of the force $F_{y}$ and perpendicular to $O P$. According to the triangle $O P Q$, the following equations are obtained:

$$
\begin{aligned}
& \sin \beta=\frac{(\mathrm{H}+\mathrm{S} / 2)}{\sqrt{L^{2}+(\mathrm{H}+S / 2)^{2}}} \\
& \mathrm{op}=\sqrt{L^{2}+(\mathrm{H}+S / 2)^{2}}
\end{aligned}
$$

Hence, the torque $T$ can be calculated as 


$$
\mathrm{T}=\mathrm{op} \times F_{y 1}=F_{\mathrm{y}} \times \sin \beta \times o p=F_{y}(\mathrm{H}+\mathrm{S} / 2)
$$

where $\beta$ is the torque angle of cantilever in the vertical direction.

Moreover, there is no torque generated by $F_{y 2}$ which is along with the other component of force $F_{y}$. When the probe acts on the cell, the force $F_{y}$ in the $Y$ axis direction will make the cantilever bend in the vertical plane, and it can be calculated by

$$
F_{z} L+F_{y}(\mathrm{H}+S / 2)=k \delta L
$$

where $L$ is the length of cantilever, $k$ is the force constant of cantilever, and $\delta$ is the vertical deflection of cantilever induced by the force $F_{y}$.

When the probe acts on the cell, the cantilever will be deflected and detected by the position-sensitive detector (PSD) through the optical lever collecting the reflected laser signal. The PSD will output two signals. One is the updown signal in the vertical direction representing the deflection of cantilever in the $Z$ direction, and the other is the left-right signal in the horizontal direction representing the twisting deflection of cantilever. Then the offsets can be expressed as

$$
\begin{aligned}
& \delta=k_{v} S_{v} \\
& \theta=k_{h} S_{h}
\end{aligned}
$$

where $k_{v}$ and $k_{h}$ are the deflection and torsional sensitivities of the optical lever detection system, $S_{v}$ is the vertical signal output, and $S_{h}$ is the horizontal signal output of PSD. A large displacement of the light spot introduced by the optical lever can be detected using the PSD. Since the light intensity distributions are different, the deflections of cantilever can be measured through the differential signals from the up-down and left-right outputs.

When the cellular shear adhesion force reacts on the probe tip, the deflection of cantilever can be measured by $P S D$. Assume that the sample is pushed away from the substrate by the cantilever with the angle $a$, the relationship between $F_{x}$ and $F_{y}$ can be expressed as

$$
F_{x}=F_{y} \tan \alpha
$$

With the modeling of the manipulation of cells under pushing, the 3-D force can be obtained. Hence, the resultant force of cantilever $F$ can be calculated by

$$
F=\sqrt{F_{x}^{2}+F_{y}^{2}+F_{z}^{2}}
$$

In order to realize the accuracy acting on the manipulation, the relation constants in each equation need to be 
obtained and calibrated. Firstly, a standard grating is used to calibrate $k_{v}$, and the relationship between the PSD vertical signal and the vertical deflection of cantilever. Moreover, a hard probe with the spring constant of 30N/m and the length of $129 \mu \mathrm{m}$ was used in this experiment to avoid the influence of ambient noise. According to equation (6), the parameter $k_{v}$ can be calculated based on the linearly regressed fitting, and here $k_{v}=72.6 \pm 3.9 \mathrm{~nm} / \mathrm{V}$.

Secondly, when the vertical deflection angle equals to the twisting angle, the PSD signal outputs should be equal. Moreover, the two angles are so small that $\theta$ is approximately equal to $\tan \theta$. Hence, the relationship between $k_{v}$ and $k_{h}$ can be expressed as

$$
\frac{\delta_{z}}{l}=\frac{k_{v} S_{v}}{l}=k_{h} S_{h}
$$

where $l$ is the cantilever length used in the calibration experiment. For the PSD with the same sensitivity in both deflections, it can be calculated as $k_{h}=k_{v} / l$. Hence, $k_{h}=0.56 \pm 0.03 \times 10^{-4} \mathrm{rad} / \mathrm{V}$.

According to the thermal noise measurement method, the thermal energy calculated from the absolute temperature is equal to the energy measured from the oscillation of the cantilever. The frequency measured in the experiment is used to calculate the spring constant as [18]

$$
k=\frac{f^{2} \rho L H}{0.1043}
$$

where $k$ is the spring constant, $\rho$ is the density of the cantilever material, and $f$ is the measured resonant frequency. Here, $k=0.6 \mathrm{~N} / \mathrm{m}$. According to equation (8), the force value can be calculated, and the cellular shear adhesion force between the cell and the substrate can also be obtained by the force modeling of the cell described in the following.

When the probe acts on the cell, it has a certain distance to the substrate. Thus, the deformation of the cell mainly occurs in the upper domain of its surface. Considering the stress relaxation phenomenon of the cell, the cellular shear adhesion force can be approximately equal to the horizontal component of the cantilever twist force, and the force is applied to the cell. The relation between the cellular shear adhesion force and the horizontal component of resultant force of cantilever can be expressed as

$$
F_{c}=F_{h}-F_{z} \cdot \eta
$$


where $F_{h}=\sqrt{F_{x}^{2}+F_{y}^{2}}$ is the horizontal component of resultant force of cantilever. $\eta$ is the friction coefficient between the cell and the non-coated substrate. It is calculated by each two measurements of cellular shear adhesion force due to the correlation of the area of cells and $F_{c}$, here $\eta \approx 0.9$.

As the pushing force acts only on the cell parts close to the probe and the probe displacement is zero in the $Y$ direction, the pushing force is considered too far to affect the measurement of cellular shear adhesion forces. In this case, the friction between the probe and the cell is neglected.

If the cell is further pushed away from the substrate surface, the shear adhesion force will cause the cantilever twist in the opposite direction until it is completely detached from the surface, and there are no additional forces acting on the $Z$ axis since the probe is non-contact with the substrate during the manipulation. Hence, according to the superposition principle of forces, when the horizontal component of the cantilever twist force equals to the cellular shear adhesion force, there is a balance between the forces on the cell-probe.

In this method, the cell samples at 30\%-40\% of cell confluence were collected for the measurement of cells. A self-made AFM system was used to implement the measurement of the cellular shear adhesion force under the cultured environment. The scanning rate was $0.4 \mathrm{~Hz}$ and the image size was $512 \times 512$ pixels obtained from the contact mode.

\section{Experiments and results}

\section{Cell imaging and positioning}

After the cell manipulation was modeled, the motion of cells was viable once the minimum pushing force was applied. The samples in the experiment were SW480 human colon cancer cells grown on an unsupported cover-slip in culture. Initially, an inverted microscope was used to observe cancer cells. Then, the probe was moved to the selected location showing only the non-overlapped cells in the scanning area, avoiding any damage of single cells in the manipulation. Figure 2 shows the topographical images of SW480 cells without and with TRAIL (Figures 2A and 2E) and their cross section plots (Figures $2 \mathrm{C}$ and $2 \mathrm{G}$ ). The experiments reveal a phenomenon in the measured topography of two cells. The finding indicates that the cells treated with TRAIL show the round shapes, and the apoptosis of SW480 is gradually appeared. The dimensions of the cells treated with TRAIL are smaller than those of the cells without TRAIL. Moreover, there might be some proteinaceous adhesive secretions on the cell surfaces and 
interfered with the cell imaging. Hence, the edge fuzzy phenomenon exists in the cell scanning. As shown in Figures 2C and 2G, and the highest point of the cell can be observed. An appropriate pushing location along the highest line was selected in this study. The histograms of elastic modulus-frequency count distributions obtained by the tapping mode are shown in Figures 2D and 2H. It clearly shows that the elastic modulus of SW480 cells without TRAIL is much smaller than that of the cells with TRAIL. The surfaces of cells with TRAIL become more stiffness and this indirectly reflects that the viability of SW480 cells with TRAIL will be reduced [20].

\section{Cell manipulation with different working speeds}

In the cell manipulation, the probe was set to a distance of about $1 \mu \mathrm{m}$ from the substrate. According to the Saint-Venant's Principle [21], when the manipulation was far away from the load acted area, the stress would be close to zero. Hence, the complex detachment process was simplified in this case, and the horizontal component of the cantilever twist force was approximately equal to the cellular shear adhesion force when there was a distance between the manipulating position and the substrate. The image of cell populations captured by $C C D$ is shown in Figure 3. The cells under the probe were used for scanning and manipulation by AFM, and those cells were independently grown on the unsupported cover-slip and easily manipulated. After the manipulation, the cell was separated from the substrate and floated in the culture indicated by the arrow, as shown in Figure 3B. Then, the maxima of probe deflections both in the up-down and left-right directions were selected and calculated by the manipulation model established above, as shown in Figure 4. Since the living cell could response to mechanical perturbation when the $A F M$ approaching to it, different probe speeds were presented to study the influence of manipulation in this work. The probe pushed the different surfaces along the highest cross section with the piezoelectric transducer (PZT) from the right to the left direction at the speeds of $15 \mu \mathrm{m} / \mathrm{s}, 20 \mu \mathrm{m} / \mathrm{s}$ and $30 \mu \mathrm{m} / \mathrm{s}$, respectively. The cantilever was deformed in the vertical direction and the torque direction due to the effects of cellular shear adhesion force and the angle $\alpha$ between the probe lateral direction and the manipulating direction. The up-down and left-right signals detected by PSD are shown in Figure 4, and the cellular shear adhesion force can be calculated by equation (8). It can be seen from the measurements that the resultant curves become smoother with the increase of manipulation speed due to the reduced detachment time in the shear direction. The impact of cell adhesion between the cell and probe can be reduced by the increase of pushing speed. If the speed of probe is slow, the maximum deformation of probe will be disturbed by some influences such as the mechanical perturbation, the 
fluctuation of the liquid and the resistance. Then, the horizontal component of the twisted direction will present interference signals around the maximum deformation. However, the faster speed might cause the oscillation during the manipulation or damage the cell, and due to the viscosity of culture, the horizontal component of the twisted direction will generate a certain deflection in the initial state which will affect the measurement of the lateral force. Thus, the different speeds of the probe were presented to study the influence of manipulation, and a suitable manipulation speed is important to reduce the extra effect without distortion in the experiment.

\section{Cell manipulation in different locations}

According to the highly precise imaging for the cell, various locations of the cell can be manipulated on the nano-scale. Moreover, the trisection locations in the middle part and the both sides of different similar cells were manipulated respectively at the speed of $20 \mu \mathrm{m} / \mathrm{s}$, as shown in Figure 5. Among them, the cellular shear adhesion force measurements in the both sides of the cell were implemented on the same cell. It can be seen that the maximum deflection of the probe manipulated on the middle part is far greater than that of the other two sides. Since the cell was separated from the substrate and floated in the culture, the measurement in the middle side reflected the holistic shear adhesion force of the single cell. Furthermore, the cell manipulation in both sides of cells was not completely the same in the measurements. After the manipulations in both sides, the cell was not separated from the substrate due to the effect on the major part of the cell, and the force curves of the two output signals showed the difference with the manipulation in the middle part. Moreover, the measurement of the cell adhesion in the upper portion of the cell is twice as much as the measurement in the lower portion. It may reveal that the cancer cells have the metastasis tendency after cultured for 16 to 20 hours. The cell adhesion is the most important factor for the research of the cell metastasis. On the basis of mechanic research of physical translocation with a novel perspective on the metastatic process, it is important for preventing metastasis in the patients diagnosed with early cancer lesions [22].

\section{Cell manipulation for different types of cells}

Cell manipulation can not only research for the metastasis of cancer cells, but also identify the cell viability. The experimental conditions were kept the same with the cell culture during the measurement. Figure 6A shows the cellular shear adhesion force measurements of twenty SW480 cells. The cell adhesion can be quantitatively 
expressed by the shear adhesion force of the unit area. Thus, combined with the morphology character of cells on the nano-scale, the cellular shear adhesion force of the unit area can be calculated, and the adhesion changes on different surfaces can be obtained. Figure 6B shows the corresponding measurements of each cellular shear adhesion force per unit area. It clearly shows that the shear adhesion forces of unit area are performed in the same level when cells cultured in the same batch.

The experimental environment was kept to the same conditions during the culture and measurements. Figure 7 shows the average shear adhesion force measurements with three types of samples on each substrate from 20 force curves. The shear adhesion forces of cancer cells were $1.7 \pm 0.3 \times 10^{-7} \mathrm{~N}$ on the non-coated cover slip. On the other hand, the shear adhesion forces of the same type of cancer cells with the TRAIL of $300 \mathrm{ng} / \mathrm{ml}$ were $0.71 \pm 0.2 \times 10^{-7} \mathrm{~N}$ on the same cover slip, and the cancer cells with the TRAIL of $200 \mathrm{ng} / \mathrm{ml}$ were $0.87 \pm 0.2 \times 10^{-7} \mathrm{~N}$. The results show that the shear adhesion force is decreased when the cells are cultured with TRAIL, which agrees well with the previous research [23]. The proposed method of cellular shear adhesion force measurement is easy to perform and quantitatively identify the viability of single cells. Moreover, combining with the high resolution images of cells before and after cantilever manipulations, the cells of interest can be presented with more information in single cell analysis.

\section{Discussions}

Cell adhesion is a biological phenomenon to maintain the morphology structure and functions of cells. It is mainly mediated by the adhesion molecules on the cell surface involved in signal transduction and cell recognition of normal cells to maintain the integrity of tissues. In cancer cells, the changes in adhesion are important factors for the invasion and metastasis of cancer cells [15]. For the cancer cells detached from the tumor, the adhesive force is weakened, and in the case that the cancer cells attach to the endothelium and then migrate into the tissue, the adhesive force must be sufficient to overcome the hydrodynamic forces in the blood. Thus, the cancer cells are able to regulate the adhesion and implement the invasion and metastasis processes.

Other methods for measuring the cell adhesion only rely on the vertical component of force since the AFM cantilever is moved vertically to the cell. Samples with displacement in a different direction are usually not used in the analysis, and the impacts of other directional force components and pushing speeds are not discussed. Moreover, the visual feedback with an optical microscope or an environmental scanning electron microscope (ESEM) could not 
present an accurate position of the cell and obtain the morphology character at the same time. With this method, a model for the deformation of the cantilever in three dimensions was established for the forces along with the three orthogonal coordinate axes, and the probe with the elastic deformation property was placed horizontally adjacent to the cell. Based on the superposition principle and the transferability of the force, cells and probes were subjected to a critical status for the detachment through a horizontal force introduced by PZT. When the probe was twisted by the force for the detachment between the cell and the substrate, the horizontal component of the twisted force was used to calculate the cellular shear adhesion force. In the measurement, the accurate manipulation on the nano-scale can be performed based on the cell morphology, especially for the different locations of the cell manipulation which is potentially beneficial to the mechanic research of physical translocation with a novel perspective on the metastatic process. Thus, the cellular shear adhesion force per unit area can be obtained and analyzed, combined with the adhesion changes on different surfaces. It reflects the physiological state of cells more accurately in molecular level.

Furthermore, in this work, the experimental results show that the cellular shear adhesion force will be reduced when the cells are cultured with the immune expression of TRAIL, as shown in Figure 7. TRAIL expressed by in vitro can protect from tumor metastasis and suppress the growth of cancer cells. In addition, the cell indentation research also shows that the elastic modulus of SW480 cells without TRAIL is much smaller than that of the cells with TRAIL, and it reflects that the viability of SW480 cells with TRAIL will be reduced due to the declined shear adhesion force and the gradually-hardened cell surface. In the experiment, two different concentrations of TRAIL have been used to study their impacts on the shear adhesion force of cells.

In this method, the variation tendency of shear adhesion forces and the morphology changes can be performed simultaneously on the nano-scale. Before the measurement of the shear adhesion force of the cancer cell, the cell was adhered to the substrate for more than 16 hours, so that the shear adhesion force between the cell and the substrate was almost unchanged, and the measurement deviation was minimized. With this method, the cellular shear adhesion force can be obtained.

\section{Conclusions}

In this work, an $A F M$ was used to obtain the morphology character of targeted cells on the nano-scale and the cellular shear adhesion force at the same time. All the manipulations can be obtained according to the high precision imaging for the cell on the nano-scale. A cell manipulation model was established based on the probe of the 
ContGB-G type (BudgetSensors), and two types of living cancer cells were pushed by the probe in culture. All manipulations were monitored by a CCD camera. We presented the results of ten individual measurements of yield strength on two types of cancer cells, one in the native state and one with the immune expression of TRAIL that reduced the binding strength. Moreover, different pushing speeds of the probe and various locations of cells were used in the experiments to study their influences. In this study, the measurement of the cell adhesion in the upper portion of the cell is different from that in the lower portion. It may reveal that the cancer cells have the metastasis tendency after cultured for 16 to 20 hours, which is important for preventing metastasis in the patients diagnosed with early cancer lesions. This method provides a way for the measurement of the horizontal shear adhesion force between the cell and the substrate, and for the simultaneous exploration of shear adhesion forces of cancer cells using the AFM imaging and manipulation.

\section{Acknowledgments}

This work was supported by International Science and Technology Cooperation Program of China (No.2012DFA11070), EU FP7 (BioRA No.612641), EU H2020 (FabSurfWAR No.644971), Jilin Provincial Science and Technology Program (No.201215136, No.20140414009GH, No.20140622009JC and No.20160623002TC), and Science and Technology Program of Changchun City (No.11KP04). 


\section{References}

[1] Ko, T. J., Kim E., Nagashima S., et al. (2013) Adhesion behavior of mouse liver cancer cells on nanostructured superhydrophobic and superhydrophilic surfaces. Soft Matter, 9(36), 8705-8711.

[2] Yeung T., Georges P. C., Flanagan L. A., et al. (2005) Effects of substrate stiffness on cell morphology, cytoskeletal structure, and adhesion. Cell Motility and the Cytoskeleton, 60(1), 24-34.

[3] Carey S. P., Charest J. M., Reinhart-King C. A. (2011) Forces during cell adhesion and spreading: implications for cellular homeostasis. Cellular and Biomolecular Mechanics and Mechanobiology, 4, 29-69.

[4] Noy A., Friddle R. W. (2013) Practical single molecule force spectroscopy: how to determine fundamental thermodynamic parameters of intermolecular bonds with an atomic force microscope. Methods, 60(2), 142150.

[5] Whited A. M., Park P. S. H. (2014) Atomic force microscopy: a multifaceted tool to study membrane proteins and their interactions with ligands. Biochimica et Biophysica Acta (BBA)-Biomembranes, 1838(1PtA), 5668.

[6] Huang Y. X. (2005) Cell biomechanics. Physics (China), 34(6), 433-441.

[7] Morkvėnaitė-Vilkončienė I., Ramanavičienè A., Ramanavičius A. (2013) Atomic force microscopy as a tool for the investigation of living cells. Medicina (Kaunas), 49(4), 155-164.

[8] Evans E. A., Calderwood D. A. (2007) Forces and bond dynamics in cell adhesion. Science, 316(5828), 11481153.

[9] Haimovitz-Friedman A., Falcone D. J., Eldor A., et al. (1991) Activation of platelet heparitinase by tumor cellderived factors. Blood, 78(3), 789-796.

[10] Vremec D., Hansen J., Strasser A., et al. (2015) Maintaining dendritic cell viability in culture. Molecular Immunology, 63(2), 264-267.

[11] Minelli C., Kikuta A., Tsud N., et al. (2008) A micro-fluidic study of whole blood behaviour on PMMA topographical nanostructures. Journal of Nanobiotechnology, 6(1), 1-11.

[12] Chen S., Cheng J., Kong C. W., et al. (2013) Laser-induced fusion of human embryonic stem cells with optical tweezers. Applied Physics Letters, 103(3), 033701-033701-4. 
[13] Picas L., Milhiet P. E., Hernández-Borrell J. (2012) Atomic force microscopy: A versatile tool to probe the physical and chemical properties of supported membranes at the nanoscale. Chemistry and Physics of Lipids, 165(8), 845-860.

[14] Lee C. C., Wu C. C., Su F. C. (2004) The technique for measurement of cell adhesion force. Journal of Medical and Biological Engineering, 24(1), 51-56.

[15] Shen Y., Nakajima S., Kojima S., et al. (2011) Single cell adhesion force measurement for cell viability identification using an AFM cantilever-based micro putter. Measurement Science and Technology, 22(11) 115802-115809(8).

[16] Maciaszek J. L., Partola K., Zhang J., et al. (2014) Single-cell force spectroscopy as a technique to quantify human red blood cell adhesion to subendothelial laminin. Journal of Biomechanics, 47(16), 3855-3861.

[17] Werneburg N. W., Eugenia G. M., Bronk S. F., et al. (2007) Tumor necrosis factor-related apoptosis-inducing ligand activates a lysosomal pathway of apoptosis that is regulated by Bcl-2 proteins. Journal of Biological Chemistry, 282(39), 28960-28970.

[18] Butt H. J., Cappella B., Kappl M. (2005) Force measurements with the atomic force microscope: technique, interpretation and applications. Surface Science Reports, 59(1), 1-152.

[19] Hoogenboom P. C. J., Spaan R. (2005) Shear stiffness and maximum shear stress of tubular members. The Fifteenth International Offshore and Polar Engineering Conference. International Society of Offshore and Polar Engineers, 316-319.

[20] Targosz-Korecka M., Biedron R., Szczygiel A. M., et al. (2012) Stiffness changes of tumor HEp2 cells correlates with the inhibition and release of TRAIL-induced apoptosis pathways. Journal of Molecular Recognition, 25(5), 299-308.

[21] Mises R. V. (1945) On Saint-Venant’s Principle. Bulletin of the American Mathematical Society, 51(8), 555562.

[22] Chaffer C. L., Weinberg R. A. (2011) A perspective on cancer cell metastasis. Science, 331(6024), 1559-1564.

[23] Targosz-Korecka M., Biedron R., Szczygiel A. M., et al. (2012) Stiffness changes of tumor HEp2 cells correlates with the inhibition and release of TRAIL-induced apoptosis pathways. Journal of Molecular Recognition, 25(5), 299-308. 


\section{Figure Legends}

Figure 1 - Modeling of a cantilever-tip in three dimensions. (A) AFM cantilever deformation model in three dimensions. (B) Model of $F_{y}$ action on the cantilever. (C) SEM lateral view of the AFM tip used for the single cellular shear adhesion force measurement. (D) SEM top view of the AFM cantilever, and the diameter of cantilever tip is less than $10 \mathrm{~nm}$.

Figure 2 - Nano-scale topography of a SW480 colon cancer cell obtained by AFM in culture. (A) Topography image of two SW480 cells, obtained by the contact mode, and the area of cell is about $416.25 \mu \mathrm{m}^{2}$. (B) 3D topography image of the cells. (C) Cross section plot of a single cell in the upper part of figure. It is oval in shape, $27.04 \mu \mathrm{m}$ long, $19.60 \mu \mathrm{m}$ wide and $3.2 \mu \mathrm{m}$ high. (D) Histograms showing the elastic modulus-frequency count distribution of SW480 cells from 136 force curves by the quantitative imaging mode, and the mean standard deviation of the elastic modulus is $352.08 \pm 241.81 \mathrm{~Pa}$. (E) Topography image of a single SW480 cell with TRAIL, obtained by the contact mode, and the area of cell is about $254.47 \mu \mathrm{m}^{2}$. (F) 3D topography image of the cell. (G) Cross section plot of the single cell. The cell in the upper part of figure is round with the radius of $14.34 \mu \mathrm{m}$ and 4.3 $\mu \mathrm{m}$ high. (H) Histograms showing the elastic modulus-frequency count distributions of SW480 cells with TRAIL from 131 force curves by the quantitative imaging mode, and the mean standard deviation of the elastic modulus is 1121.59 $\pm 943.54 \mathrm{~Pa}$.

Figure 3 - The image of cell populations captured by $C C D$. (A) $C C D$ image of cell populations before manipulation. The AFM tip was moved to the target single cell. (B) CCD image of the cell population after manipulation.

Figure 4 - Experimental results. (A) The left-right signal detected by PSD when the probe speed is $15 \mu \mathrm{m} / \mathrm{s}$. (B) The up-down signal detected by PSD when the probe speed is $15 \mu \mathrm{m} / \mathrm{s}$. (C) The left-right signal detected by PSD when the probe speed is $20 \mu \mathrm{m} / \mathrm{s}$. (D) The up-down signal detected by PSD when the probe speed is $20 \mu \mathrm{m} / \mathrm{s}$. (E) The left-right signal detected by PSD when the probe speed is $30 \mu \mathrm{m} / \mathrm{s}$. (F) The up-down signal detected by PSD when the probe speed is $30 \mu \mathrm{m} / \mathrm{s}$. 
Figure 5 - Experimental results. (A), (B) and (C) were the left-right signal and up-down signal detected by PSD when the cell was manipulated in the middle part, and the schematic diagram of the manipulation, respectively. (D), (E) and (F) were the left-right signal and up-down signal detected by PSD when the cell was manipulated in relatively slender part on one side, and the schematic diagram of the manipulation, respectively. (G), (H) and (I) were the left-right signal and up-down signal detected by $P S D$ when the cell was manipulated in relatively large part on the other side, and the schematic diagram of the manipulation, respectively.

Figure 6 - Cellular shear adhesion distributions. (A) The cellular shear adhesion force measurements of twenty cells. (B) The corresponding measurements of each cellular shear adhesion force per unit area.

Figure 7 - Average shear adhesion force measurement with the deviation of two types of samples on each substrate. 